\title{
1. Introduction to The Policy Uptake of Citizen Sensing
}

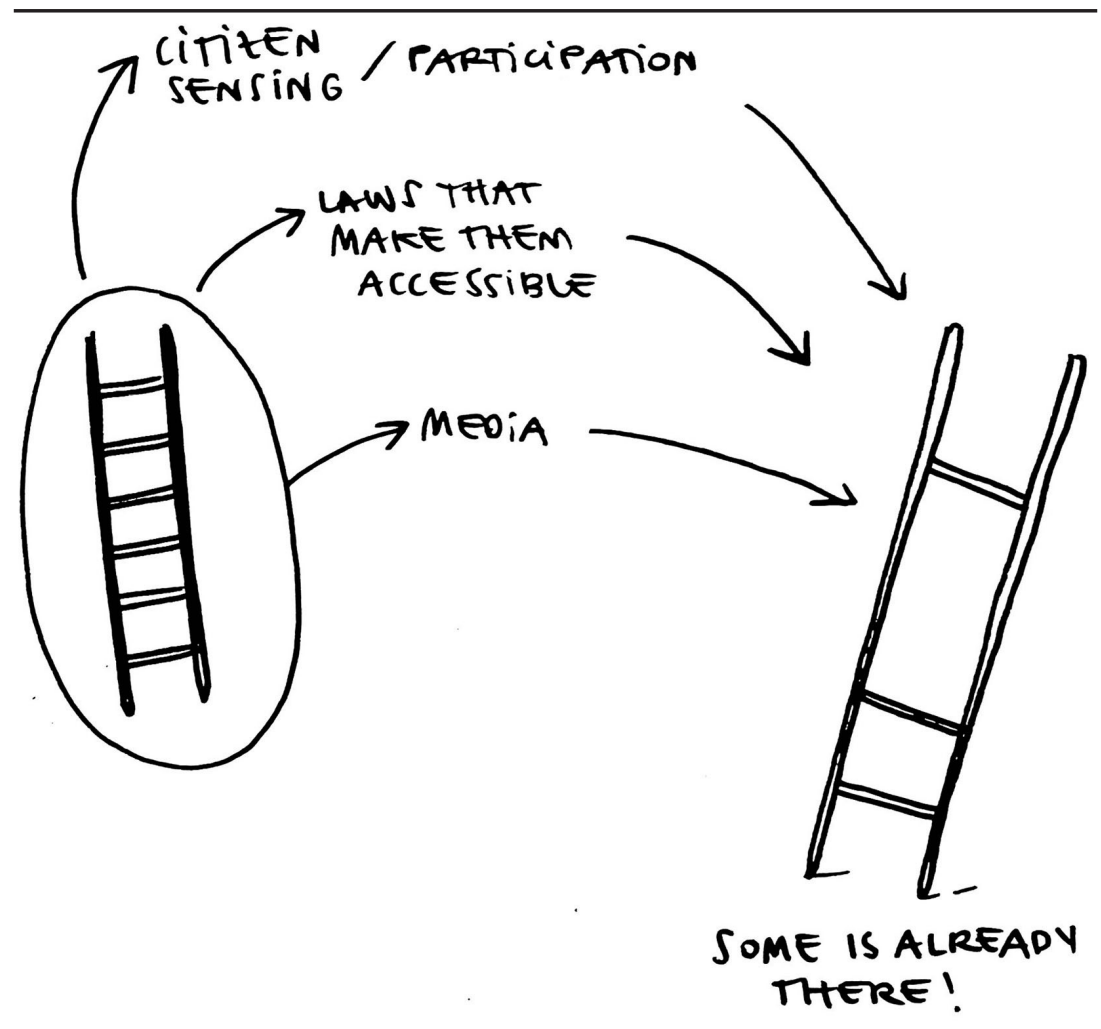

Source: $\quad$ Alice Toietta for this project, 2020.

Figure 1.1 Researching citizen sensing in relation to law and policy

\subsection{A PRESSING INQUIRY}

As environmental governance scholars note, 'demands for accountability continue because accountability procedures do not respond to environmental stakeholders' expectations (those affected by environmental problems and 
those that claim to speak for the environment)' [emphasis added] (Kramarz and Park 2016, 5). Through this study, I aim at investigating a response to this problem of (perceived) legitimacy and accountability gaps in the governance of environmental risks to public health. The 'expectations' here considered are those of communities and individuals that are or feel to be exposed to environmental risks endangering their health.

The response to these expectations takes the form of a technology-mediated practice, that of 'citizen sensing', here defined as a form of grassroots-driven monitoring of initiatives aimed at tracking environmental factors (in alternative or in addition to official governmental monitoring), making use of Information and Communication Technology (ICT), in general, and, in particular, of sensors. I frame citizen sensing as a technology, a social phenomenon and a method as well as a form of (environmental) rights in action, as Chapter 2 will further describe. I inquire whether practices of citizen sensing can be (part of) a possible solution to the mentioned accountability and legitimacy gaps in institutional (environmental) risk governance, through the uptake by institutional actors of such grassroots monitoring initiatives.

In the book The Paradox of Scientific Authority, Bijker, Bal and Hendriks $(2009,6)$ showed how participation by citizens (in public and environmental health governance) is increasingly complementing the 'old institutional mechanisms of democracy'. This book is situated exactly at the 'crossroads between science and politics' where 'the democratic governance of technological cultures' takes place (Bijker, Bal and Hendriks 2009, 23). I understand this latter notion as the 'appropriation' by citizens of technology to express claims to a healthier environment and to access environmental information, as well as to make environmental risk governance more open and accountable (Berti Suman 2020a).

This study targets the still unanswered yet urgent question on what makes government agencies use citizen sensing data and methods. It is also a reflection on how these institutional actors are using, could or should use the citizen sensing information that is deployed. This inquiry is increasingly important for a number of reasons, including the rapidly expanding availability of low-cost sensing devices in the hands of lay citizens, the continuing resource constraints that government agencies face, the information gaps in existing government-based sensing programmes for environmental monitoring, which often leave toxic exposures (particularly in environmental justice communities) undetected, and the new constraints and challenges that governments experience in these times of an exacerbated pandemic.

The COVID-19 'storm' offered and is offering an opportunity to rethink the allocation of responsibilities between citizens and institutions, and among institutions. It also compels authorities to adopt policy changes oriented to a more horizontal governance of environmental and health challenges, which 
may eventually deemphasize traditional top-down enforcement to the benefit of a greater civic responsibilization. Lastly, in pandemic times, governments need to find creative pathways to restore civic trust in institutional processes; listening to citizen sensing could be part of the story. ${ }^{1}$

\subsection{STATE OF KNOWLEDGE}

Studies on the implications of citizen sensing practices for governmental processes and interventions are scarce to date. Craglia and Shanley $(2015,690)$, partially along this line, defend the societal and policy benefits of 'opening up the bases of decisions by government agencies and private corporations [to contributions from] citizen science'. Misuraca and Pasi (2019), more broadly discussing the potential of ICT-enabled social innovation, note that it can constitute a resource for governments, supporting the provision of better and more efficient social services and increasing the well-being of citizens. Literature on public participation in (local) governmental processes (Michels and De Graaf 2010; Kelty 2017), however, has also highlighted the challenges of interfacing grassroots and institutional actors, and the shortcomings that policy-makers and citizens experience when governments 'adopt' or 'support' citizen initiatives. Instead, scant attention has been devoted to exploring such experiential outputs in the case of citizen sensing and to the implications that this may have for designing and governing participatory processes. This book will show that the drawbacks of policy adoption also exist for citizen sensing, and should be carefully considered when pushing for policy uptake. However, my analysis will also demonstrate that successful (yet very context-dependent) instances of policy adoption are possible, and it will reflect on the factors underpinning this outcome, in order to guide governmental responses to citizen sensing.

Drawing on risk governance theories and taking (mostly) the perspective of European laws and policies, this book thus targets the policy dimension of citizen sensing. The topic is highly relevant for a number of reasons, but has especially received a high momentum now as risk governors around the world - in addition to a growing number of civil society actors - are turning to citizen sensing initiatives as a potential source of precious evidence, especially in disaster and crisis scenarios. While this book was being finalized, numerous countries around the world were witnessing the emergency associated with a massive, systemic risk, i.e. the COVID-19 pandemic. Citizen science and sensing initiatives are multiplying in response to the crisis, both to offer relief

\footnotetext{
1 See on this aspect a blog post written with Marie Petersmann https://blog.uvt.nl/ environmentallaw/?p=430. Accessed 15 November 2020 .
} 
to affected people and to provide resources for policy-makers and scientists. ${ }^{2}$ The perception that the risk is not (always) being properly handled and communicated by competent authorities is making citizens respond to uncertainty and fear with creative initiatives, which ultimately are a demonstration that people wish to retain agency in times of emergency. ${ }^{3}$

Beyond the pandemic, since I started engaging with this research, a number of transformations drastically changed the scene. Citizen sensing communities are having their data recognized in environmental litigation, as observed in the Formosa case decided on July 2019. ${ }^{4}$ A new Citizen Science Law and Policy Working Group ${ }^{5}$ is mobilizing researchers around the world to inspect legal and policy questions associated with citizen sensing. Legal scholars are gradually joining the debate too. ${ }^{6}$ Moreover, the European Commission just published guidelines ${ }^{7}$ that represent a first step towards a regulatory instrument for stimulating the use of citizen science for European environmental policy. Also recently, on the Joint Research Centre data catalogue, an expanded version $^{8}$ of the inventory of environmental citizen science projects for policy has been released, giving new momentum to the data set and related discussions. Conceivably, discussions in the years to come will boost the relevance of the field and make this book particularly appealing. ${ }^{9}$

2 For example, in Italy, the project 'Covid19italia.help', was launched as a citizen initiative to 'sense' and collect information related to the virus, in order to make it easily accessible for people in need and competent institutions. See https:/www .covid19italia.help/about/. Accessed 15 October 2020. Numerous other initiatives are spreading around the world. See for a collection of ongoing projects http://eu-citizen .science/citizen-science-resources-related-to-the-covid19-pandemic/. Accessed 26 October 2020.

3 Intervention by Professor Ortwin Renn, Institute for Advanced Sustainability Studies, IAAS Postdam, Germany, at SHARE Webinar: 'Lessons we are learning from the COVID-19 pandemic for radiological risk communication', 16 March 2020. See https://www.ssh-share.eu/webinar/. Accessed 26 March 2020.

4 USA District Court, Southern District of Texas, San Antonio Bay Estuarine Waterkeeper et al. v. Formosa Plastics Corporation et al. [2019] No. 6:17-cv-00047.

5 See https://www.citizenscience.org/working-groups/law-policy-working-group/. Accessed 15 March 2020.

6 For example, within the framework of the recently launched 'PANELFIT project - Participatory Approaches to a New Ethical and Legal Framework for ICT', targeting also citizen science and sensing. See https://www.panelfit.eu/. Accessed 15 March 2020.

7 European Commission 2020.

8 See https://data.jrc.ec.europa.eu/dataset/jrc-citsci-10004/resource/e9a6cbb2-a3 f5-46c3-8bc1-7fd94d600d0f. Accessed 2 April 2020.

$9 \quad$ I am currently contributing to these discussions through my ongoing Dutch Research Council (NWO) Rubicon project that started in summer 2020 and that will develop in a Marie Curie Individual Fellowship on the project titled 'SENSJUS - 


\subsection{FROM THE KNOWLEDGE GAP TO THE RESEARCH QUESTION}

In short, this book contributes to fill the outlined knowledge gap by targeting the following question: 'Which factors contribute to the policy uptake of community-led citizen sensing, responding to a risk and eventually generated by distrust?' The question mainly addresses the policy-makers' side, exploring what makes them receptive to the inputs from the 'sensing citizens' through a 'meaningful policy uptake'. A meaningful policy uptake is here defined as 'the adoption by institutional actors of (some component of) the initiative and/or the performing of policy/regulatory/factual interventions expressly demanded by the initiative or, in any event, stimulated by the initiative'. Policy uptake is characterized as 'meaningful' in the sense that institutional recognition and adoption should concretize in actions aimed at mitigating or removing the risk at issue, where it is possible, or at better handling it. The actual removal or mitigation of the risk, however, is not assessed in this book.

To answer this question, in a first stage, I reach a working definition of citizen sensing and isolate four elements of an initiative that I consider relevant for this research: the technology element, the grassroots' drive, the risk element and the sense of distrust from the citizens towards the responsible institutions. Afterwards, I demonstrate that citizen sensing needs recognition through uptake, which may occur through both social and/or policy uptake. I thus argue that citizen sensing needs both social and policy uptake to be effective, although I acknowledge that this can sound paradoxical, especially for the second uptake. However, while it is true that the sensing citizens act in response to governmental failures, I will show that they still have to rely on institutions to fully reach their goals in terms of removal, mitigation or better handling of the risk at issue. Thus, a 'need for policy uptake' will emerge. Having affirmed that, I empirically research whether and how the policy uptake of citizen sensing occurs in practice, and I assess the influence of the four key factors plus social uptake on this outcome.

The four elements, three of them working as 'preconditions' for an initiative to be considered for the aims of this research and the other relevant for the outcome but not indispensable, are derived from my exploratory empirical analysis of the field and are framed through theoretical concepts extracted from

Citizen Sensing as a source of evidence in environmental justice litigation and as a tool for environmental mediation'. See https://sensingforjustice.webnode.it/. Accessed 26 November 2020. 
review of the relevant literature. They are translated into 'constructs', ${ }^{10}$ which are: 'Grassrootness', capturing the extent to which the initiative is driven by civil society actors; 'Riskness', identifying the extent to which the problem that the initiative tackles is serious; 'Sensorness', representing the extent to which the initiative relies on sound technology, produces valid data and adopts effective data visualization and dissemination strategies; and 'Distrust', expressing the individual and collective distrusting discourse towards the authorities competent for managing the risk at issue. These four elements are empirically researched, targeting their (conceivable and actual) influence on policy uptake.

The study on the influence of the four outlined elements on policy uptake is complemented by the analysis of an additional element, the social support ('social uptake') that the initiative receives, which may influence the policy uptake but can also be influenced by the first four elements discussed. Considerable social uptake of citizen sensing is defined as 'the outcome of the initiative gaining visibility on public media and the increase over time of the citizen sensing group'. As a result of the social uptake, citizens may adjust their actions and adopt certain conducts which could eventually lead to mitigation or even removal of the risk exposure. This could (arguably) mitigate the need for policy uptake. Social uptake, however, is here considered only to the extent that it stimulates (or hinders) policy uptake.

The aim of this book 'in a sentence' would be about the assessment of how technology, the grassroots-drive, the risk element and distrust from the sensing citizens towards the competent institutions influence the policy uptake of citizen sensing. The hypothesis which has been developed based on existing literature and on exploratory empirical analysis, and which will be tested through a combination of empirical methods, posits that these factors do have a certain influence on the studied phenomenon. From the findings of the hypothesis testing, an integrative framework for stimulating such a policy uptake (including a reflection on the 'dilemma of integration') has been extracted in a separate manuscript, an accessible booklet for interested scholars and communities (Berti Suman 2020e).

Overall, this research may be viewed as belonging to the basic or fundamental research domain (Swanborn 2009, 13). Nonetheless, some of my findings can have applications for policy-makers, practitioners, and citizen sensing communities. The queries developed and answered in this book are, in part, exploratory in the sense that they inspect in an open-ended manner the stance

10 It should be noted that these words used to refer to the constructs are neologisms that I decided to create as existing words (such as 'Riskiness') had a different meaning than what I wanted to convey with my constructs. By using neologisms, I could capture and express the meaning that this study gives to each construct. I will refer to them in italics to stress their nature of neologisms. 
of citizen sensing in specific fields (i.e. risk governance and environmental law). Some are instead explanatory as they aim to understand which factors influence a certain outcome. Other questions are descriptive, aiming to define the practice or the studied outcome. A design effort - aimed at drawing an integrative framework for citizen sensing to structurally contribute to risk governance - is instead offered in Berti Suman 2020e.

\subsection{METHODOLOGY AND METHODS}

This research is based on a combination of an inductive and a deductive approach. I first shaped my theoretical frame on (exploratory and casuistic) empirical data and, at a later stage, tested my (theoretical-informed) hypotheses in the empirical reality. I started exploring my research field using concepts from theory (i.e. 'sensitizing concepts', such as risk governance failures, environmental rights, accountability claims), which acted as a lens that channelled my attention to certain aspects during my exploratory empirical data collection. These sensitizing concepts were mostly defined through deductive delineations from literature and legal documents interpretation. While performing my exploratory data collection, in parallel I was indeed reviewing literature on risk governance and on environmental norms, justice and rights, as well as relevant legal documents. Adopting a constructivist approach, my understanding of citizen sensing and its key components emerged inductively throughout the empirical research, but I read them through the lens of the overarching theoretical notions referred to above. Overall, I had to engage in empirical analysis for this study due to the fact that citizen sensing is a relatively 'recent' practice that has rarely been studied in relation to risk governance, environmental rights and distrust. Thus, the data already available was not adequate or sufficient to answer my research question.

The research has been characterized by an exploratory stage, where I still had to refine my research question and sub-questions (Swanborn 2009, 61). I could thus frame the data emerging from my exploratory empirical analysis through my ongoing theoretical reflections, which - in turn - were also shaped by the preliminary insights I was obtaining in the field. On the basis of my exploratory field analysis, I structured my hypotheses and the underlining questions. I further proceeded to test them through a triangulation of methods, approaching the research object 'from different angles' (Swanborn 2009, 64), combining methods in an iterative process, where I started with a purely qualitative approach to then dive into a method bridging qualitative and quantitative analysis, and subsequently returned to the qualitative approach. I opted for a mixed-methods research approach as an avenue to address 'more complicated research questions and collect a richer and stronger array of evidence' compared to a single-method approach (Yin 2018, 63). 
Yet, I faced challenges in combining more open-ended, qualitative research modalities with the hypothesis-driven and targeted mind-set of larger-N studies. I tried to reconcile the tensions between the two approaches by 'staying adaptive' (Yin 2018, 84) and being flexible to methodological adjustments. Overall, I could find an 'encounter' between a qualitative and quantitative approach in the fuzzy-set Quantitative Comparative Analysis (fsQCA), a method combining case-oriented and variable-oriented quantitative analysis, and bringing the empirical intensity of qualitative approaches to studies of a large number of cases. In approaching this method, I had to adapt my terminology at times, for example moving from the use of terms specific to qualitative analysis, to 'conditions', 'outcome' and 'assessment of directional expectations', typical of fsQCA, which I refer to as 'variables' and 'hypothesis testing' in all chapters except for the section on the fsQCA, to adopt a more standard, known terminology.

Overall, the leading strategy that shaped this research project is the case study approach or, in other words, field research (Swanborn 2009, 93). The case study approach here not only played the role of a research strategy but it has also been the object of applications of a number of methods deployed to study selected cases. I primarily decided to adopt the case study approach in order to understand a 'real-world case' (Yin 2018, 15), i.e. the policy uptake of citizen sensing. I assumed that the understanding of this phenomenon required the analysis of 'important contextual conditions' (Yin 2018, 15). The case study method is indeed particularly helpful when the boundaries between a phenomenon of interest and its context are not clearly separable (Yin 2018, $15)$. As Yin $(2018,18)$ pointed out, a case study research 'benefits from the prior development of theoretical prepositions' to guide the data collection design and implementation, which to some degree it is true in my case, and 'relies on multiple sources of evidence [...] in a triangulating fashion', which is exactly the approach I followed in this research. Moreover, the case study approach has been selected for its aptness to monitor processes (Swanborn 2009 , 96), given that the policy uptake of citizen sensing is exactly a process. Lastly, this strategy has been selected, especially in the first phase of the research, as a way to perform an exploration of the field and identify my hypotheses and variables. Through the case study approach, I could identify the contextual characteristics of the phenomenon under study and choose 'the most relevant determinants' to the aims of my research (Swanborn 2009, 95).

A set number of citizen sensing cases (multiple case study analysis, Yin 2018) have been intensively researched in their natural context and in their development (Swanborn 2009, 92). Two of them were the object of in-depth qualitative analysis in an exploratory stage of the research; many more were included in the targeted, hypotheses-testing stage of the analysis and researched through the fsQCA method. The studied cases do not appear as separate chap- 
ters in the book, but the findings from the case study analysis are integrated in the chapters, while the core of the empirical analysis is in Chapter 4.

Overall, the data for this research have been collected primarily in the field of environmental risks to public health and of citizen science and sensing initiatives responding to these types of risk. The data collection and respective analytical strategies adopted can be summarized as follows:

- Literature review ${ }^{11}$ aimed at collecting non-elicited or secondary data ${ }^{12}$ from scientific publications, such as academic papers revolving around the topic of citizen sensing; legal, socio-political and Science and Technology (STS) scholarship on risk governance, environmental justice, co-production and the role of non-expert knowledge in society, which served the construction of the theoretical frame; and grey literature; such as white papers, reports and toolkits produced by citizen science and sensing projects, and by the USA/European Citizen Science Association ((E)CSA) and the European Commission (EC) on citizen science for environmental policy;

- Legal review on environmental law documents from national, international and European Union (EU) legislation (such as the Aarhus Convention and the Stockholm Declaration) and on regulatory frameworks for environmental risk governance;

- Case law review on relevant jurisprudence dealing with environmental rights, with environmental issues pinpointed by citizen sensing initiatives, and with the use of citizen-sensed data in courts;

- Secondary analysis of data files of earlier social research (such as prior case study research performed on existing citizen sensing projects);

- Text and network analysis of mass communication messages (such as citizen sensing related blog posts and newspaper articles), of citizen sensing related websites, and of email discussions within citizen sensing groups;

- Qualitative analysis of:

1. spontaneous behaviour in the field, observed systematically at the AiREAS premises, Eindhoven, the Netherlands; at the Safecast premises, Japan; and at the EC Joint Research Centre (JRC), Ispra, Italy, in

11 Search terms for the literature review included 'citizen science', 'citizen sensing', 'community-based monitoring', 'crowd-sensing', 'participatory science', 'public engagement', 'citizen participation', 'co-production', 'lay knowledge', 'risk governance', 'environmental risk to public health', 'environmental rights', 'environmental justice', 'environmental compliance assurance', 'environmental monitoring', 'sensor technology', 'environmental/health data sharing', 'accountability', 'legitimacy', 'distrust' etc.

12 The dichotomy elicited versus non-elicited data used to describe the data sources was inspired by Swanborn $(2009,64,86)$. 
addition to occasional observations at thematic workshops and citizen sensing encounters; ${ }^{13}$

2. responses from in-depth semi-structured interviews with key persons in the citizen sensing field and in other relevant fields, such as environmental risk governance and environmental law, and with participants and project leaders of citizen sensing initiatives;

3. responses from (exploratory and targeted) web surveys with participants and project leaders of citizen sensing initiatives, where the exploratory survey was functional to identify respondents for follow-up in-depth interviews, and the second survey was to explore the interplay of variables;

- Descriptive analysis of a large-N data set consisting of 503 cases $^{14}$ of citizen science for environmental policy developed by the JRC;

- Targeted $f_{S} Q C A$ on a data set of selected practices' extracted from the larger data set, consisting of 45 cases $^{15}$ selected in the JRC study out of the 503 cases.

Table 1.1 illustrates the main data analysed for this research project, their sources, the criteria according to which these data have been selected to be included in the study (sampling method), and how these data have been analysed throughout the research. For a detailed illustration of how I performed the qualitative research, also in terms of ethical safeguards, I refer to Annex I; ${ }^{16}$ for the descriptive analysis of the JRC data set and the text and network analysis discussed in Chapter 4, I refer to Annex II $;{ }^{17}$ for the fsQCA, I refer to Annex III ${ }^{18}$ the last two being available on a public data repository in Open Access.

13 Funding for Japan-based field research obtained from Tilburg University Special Data Collection Requests ('BAD') Fund for research projects entailing intensive data collection.

14 Data set and metadata available at http://data.jrc.ec.europa.eu/dataset/jrc-citsci -10004. Accessed 14 March 2020.

15 Data set of selected practices available at https://data.jrc.ec.europa.eu/dataset/jrc -citsci-10004/resource/31372a55-bdb5-48dc-a520-8bbf57511079. Accessed 14 March 2020.

16 Annex I - Data annex for the qualitative analysis. Available online on EasyDANS under Restricted Access at https://doi.org/10.17026/dans-262-3jwn.

17 Annex II - Data annex for the descriptive, text and network analysis. Available online on EasyDANS under Creative Commons licence at https://doi.org/10.17026/ dans-zre-t3hd.

18 Annex III - Data annex for the fuzzy set qualitative comparative analysis. Available online on EasyDANS under Creative Commons licence at https://doi.org/10 $.17026 /$ dans-xfs-qmgn. 


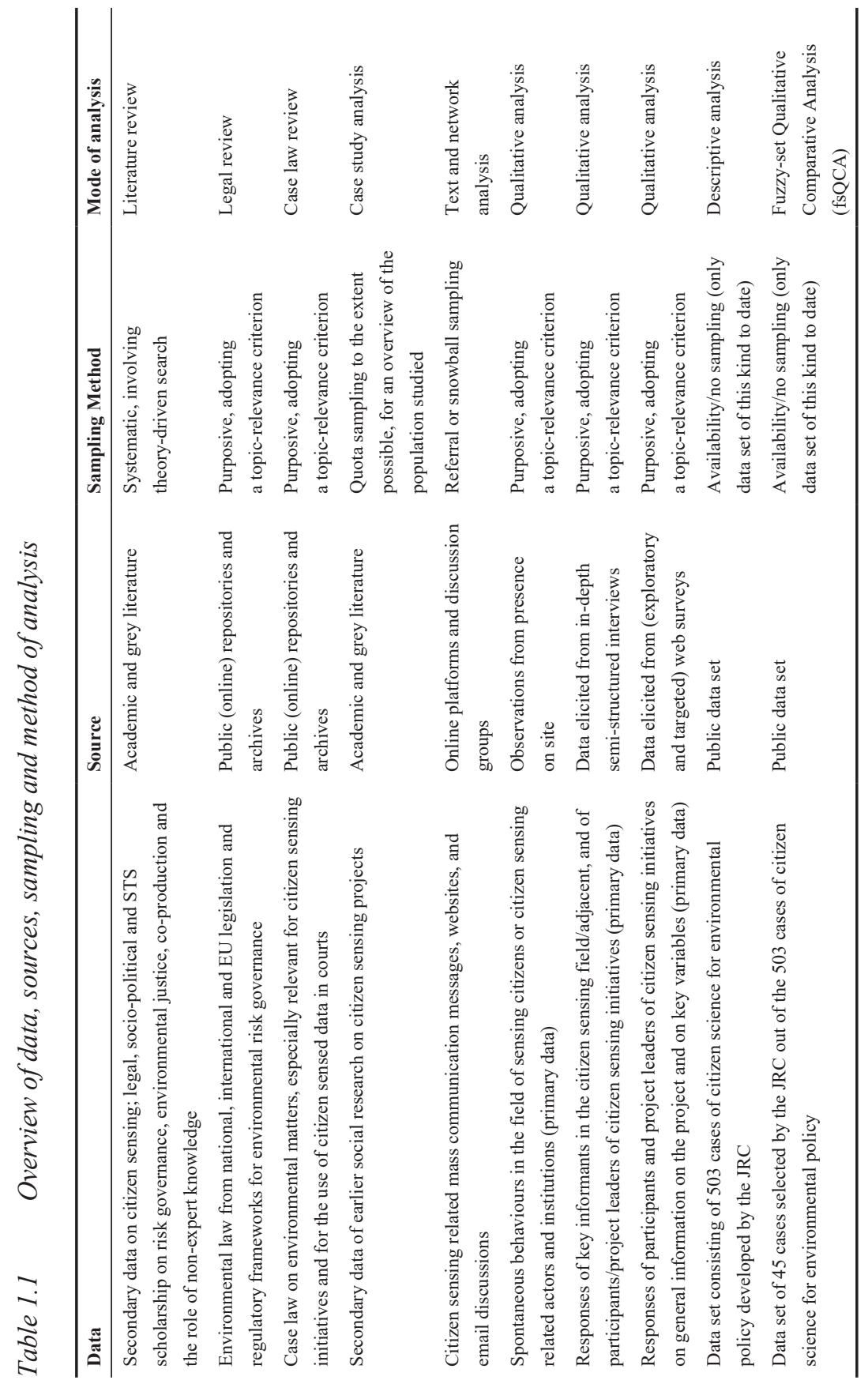




\subsection{SETTING THE SCENE: A FEW DISCLAIMERS}

Before entering the substance of this research in the following chapters, I wish to stress some points. First, the geographical scope of this study is mostly European. In consideration of the fact that the citizen sensing cases analysed here are primarily located in Europe (with minor but relevant exception such as the Safecast case, Japan, which, however, soon became an international phenomenon), the geographical area of interest for this analysis is mostly Europe and the European risk governance and legislative scenario. However, where appropriate, insights from other countries and jurisdictions (e.g. the USA and Japan) have been included in the analysis.

Moreover, among the broad panorama of citizen sensing manifestations, the narrower focus on those citizen sensing practices aimed at monitoring environmental risks that affect public health is adopted. The interplay between the governance of environmental risk to public health and citizen engagement in the tracking thereof will be presented as an outstanding example of the conflict between expert and laypersons' knowledge in facing complex risks. This conflict will be presented as an (often) necessary stage to generate a dialogue between the institutions and the people, develop mutual trust and ultimately reach a shared solution.

Furthermore, another selection criteria should be accounted for here, which is the initiation of the practice by grassroots groups. The citizen sensing initiatives considered in this research are (primarily) launched by non-governmental actors that are not yet organized in institutionalized entities. Rather, they are initiated by civic collectives formed spontaneously in response to a risk problem. This narrow choice is justified by the fact that the present research aims at verifying whether purely grassroots-driven citizen sensing initiatives can succeed in influencing and ultimately improving risk governance, through the form of an uptake of the citizens' initiative by institutional actors. An initiation of the project coming from the government (e.g. which could provide funding, tools and administrative support) is seen as a 'game changer' of the pure citizen-driven dynamics. In addition, the idea of targeting citizen-initiated forms of sensing is inspired by the aim of looking at those projects that tackle the real (or at least perceived as real) needs of the actors exposed to an environmental risk, often also strengthened by a sense of distrust towards institutions. Instead, citizen sensing projects initiated by institutional actors may represent the implementation of a political agenda that does not always match the demands and expectations of citizens. Clearly, a different angle could have brought interesting results too. However, in this research, the starting point is that the initiative begins free from institutional influences and can be considered 'purely' grassroots-driven. 
In addition, this research takes mainly the perspective of citizen sensing communities and initiatives rather than that of institution-specific traits. Although I decided to start from the perspective of the sensing citizens, aware of potential pitfalls of using elicited data, the risk of research bias and closeness to reality (Swanborn 2009, 65), I tried to limit my engagement in the research field. For example, I intentionally decided not to take part in any of the citizen sensing projects studied as a 'sensing citizen' and did not affiliate myself with the (civic) organizations that entered my study, in order to maintain a sense of distance and objectivity. Yet I was very much confronted with the division or divide between science and non-science as I engaged with the research field. Although citizen science and citizen sensing are mostly situated in the 'science' realm, they do trigger considerations that come from the non-science dimension, such as perception, trust and emotions. These considerations clearly shaped my own attitude towards the research field, the choice and the deployment of my qualitative analysis.

On a different note, I also tried to address the risk that the elicited research situation could have been artificial and 'disconnected from the real world' (Swanborn 2009, 65). To this aim, I strove to 'meet the people where they were', namely by organizing meetings, interviews or observations where the participants were usually working, living or practising their citizen sensing activities. I tried to refrain as much as possible from inserting 'extraneous' elements in the field and from shaping it with my presence. Yet, just the act of organizing a meeting or questioning, or even just being present in the field already created some sorts of deviation from the 'real life' situation that were impossible to avoid.

As a more general, maybe 'moral' consideration, also aware of my past experience, I wish to stress that there are countries where individuals reporting environmental risks and fighting against the unjust governance of such risks are silenced by powerful actors, prisoned or even killed. ${ }^{19}$ However, there are also instances where the reporting of environmental risks by citizens can happen peacefully and can even contribute to improve the handling of such risks. This is of course a niche compared to all cases of environmental conflicts. The present research consciously focuses on a 'lucky' minority of the broader group of all individuals and collectives fighting for environmental protection, i.e. those that can report and combat environmental hazards through citizen sensing practices, without risking their life. In this research,

19 In 2017, a rate of four land and environmental activists killed each week was reported by Global Witness, making that year 'the deadliest year' for this type of murder. The report was published in 2018 and it is available on Global Witness' website, see https:/www.globalwitness.org/en/campaigns/environmental-activists/ defenders-annual-report. Accessed 9 August 2018. 
I will demonstrate that not only can they effectively spread their voice through citizen sensing networks and media, but eventually they can even be listened to by those actors that are responsible for governing the risks at issue. My hope is that, in the future, the number of individuals and groups that can influence environmental risk governance (in a riskless way) will grow and the menaces for environmental defenders will decrease.

Some additional disclaimers should be made at this stage. First, there are considerable 'angle' biases in my analysis. As a matter of fact, during these years of research I have been based and travelled in and through mostly Western democracies. My understanding of key concepts such as 'citizen', 'policy-makers', 'policies', 'governance' and 'participation' has been highly influenced by this perspective. Had my research taken place in totalitarian regimes where the significance of the public sphere and of citizen participation is very different (and, participation, eventually, prohibited or challenged), my approach, lens of analysis and ultimate findings would have differed. ${ }^{20}$ This need to adapt to context-dependency resulted in the analysis of the word 'citizen sensing', which is here used in its English form as English is the language of this book. In Chapter 2, the different declinations of the term are illustrated, in view of the fact that certain countries translated the word in their language. As a caveat, it should be said that the word 'sensing' has two main meanings in English. One is related to the technical understanding of the term, standing for the act of 'detecting'. However, the word also means the act of 'feeling' or 'experiencing' something (corresponding to the German word 'ahnen': 'to suspect; to presage; to intuit'), thus including an emotional act beyond mere detecting. ${ }^{21}$ Indeed, the practice of citizen sensing can be regarded as a method of detection but it also entails an emotional engagement of the sensing actors and can trigger crucial feelings, such as a sense of anger, of distrust and - eventually - of responsibility.

Needless to say, numerous other choices and decisions have been shaped by my 'research home environment', for example which theories to consider, which cases to analyse, which criteria to adopt, which experts to contact and which participants to involve. All these choices heavily influenced the design, implementation and findings of this research. Certain aspects of the research object have been emphasized, whereas others were neglected, and all these

20 This reflection has been inspired by a discussion with Ulf Schmidt, Professor of Modern History and Director of the Centre for the History of Medicine, Ethics and Medical Humanities at the University of Kent, occurred on 7 August 2018, during my research visit at the Brocher Foundation, Geneva, Switzerland. The Brocher Foundation is a research center focused on the ethical, legal and social implications of recent medical research and new technologies.

21 Observation suggested by Ulf Schmidt, see footnote above. 
choices have 'substantive implications' (Bijker, Bal and Hendriks 2009, 39). Therefore, it is important to stress that, shifting the underlying decisions and angles, this research could have been otherwise.

In addition, I wish to underline that, from the literature analysis and the empirical research, I realized that the citizen sensing groups, despite being a form of grassroots' participation, are not necessarily equal in themselves, representative of society at large, or accessible to all lay people. These groups are nothing more than new organizational structures, with embedded social biases, power imbalances and gatekeepers. It will be stressed in this book how the majority of citizen sensing projects tend to attract 'environmentally mindful participants' who may well already be a civically engaged and privileged minority (the 'usual suspects'). However, I will also discuss the risk element as a possible trigger for broader participation beyond the 'usual suspects'.

A number of possible adverse effects of citizen sensing are not discussed here, although I acknowledge their importance. The afore-mentioned issue of the exclusion of the 'non-connected', but also issues of surveillance, network dependencies, privacy and data protection (see, for this last aspect, Berti Suman and Pierce 2018) are considered worthy of study, although they fall outside the scope of this project. However, I will address some of these concerns in tackling the barriers to policy uptake. In the final recommendations, I will also point to the need for future research along these lines.

\subsection{THE STRUCTURE OF THE BOOK}

The book unfolds along the following lines. Chapter 2 dives into the nature of the practice and its evolution. I will reach a working definition of 'citizen sensing' through which the practice is inspected in its nature of being a (working) technology and method, but also a social phenomenon and a form of 'rights in action'. The chapter will point to four elements - three preconditions for inclusion and a characteristic not a precondition - characterizing a theoretically relevant sensing initiative for the aims of this study. These four elements will be presented as the independent variables influencing the outcome that I will assess and will be 'operationalized' in measurable constructs.

Chapter 3 starts from making a case for why citizen sensing should be recognized by institutions. It provides a theoretical definition of 'meaningful policy uptake' for the aims of the research. It also engages in a theoretical analysis of a phenomenon - the social uptake of citizen sensing - that could matter for policy uptake. Literature on the two forms of uptake and their relationship is discussed and, from there, I extract my intermediary and dependent variables, and operationalize them in measurable constructs, in order to assess empirically the factors contributing to the policy uptake of citizen sensing in 
the next chapter. I will also identify possible barriers to the policy uptake from institution-specific features, as well as contextual and punctual factors hindering the institutional adoption of citizen sensing practices.

Chapter 4 illustrates the findings of an exploratory qualitative inquiry from which my hypotheses are built. Next, the hypotheses are tested, i.e. the influence on the policy uptake of the four independent variables (plus social uptake) is assessed, adopting a combination of methods, including targeted qualitative analysis, descriptive analysis of a larger data set and fsQCA. Especially from the fsQCA, it emerges that sound technology, good data quality and an effective data visualization are almost 'condicio sine qua non' to obtain the interest of policy-makers. In addition, the fact that the initiative responds to a pressing risk mismanaged by the competent authorities particularly facilitates the policy uptake. Lastly, unexpectedly, the fsQCA shows that a distrusting discourse from the sensing citizens towards the institutional handling of the risk seems a triggering factor for governmental adoption. In conclusion, I argue that there seems to be a trade-off between social and institutional support.

In the Conclusion chapter, I summarize the findings of this study and their implications, especially by illustrating the main factors that proved to be favourable for the policy adoption of citizen sensing. I will also acknowledge the emergence of an 'integration dilemma' in the attempt to integrate into governmental structures a practice that is, by nature, grassroots-driven. Lastly, I will make suggestions for a future research agenda by identifying retrospectively some limitations of this research. 